Bitte beachten Sie: Aufgrund der Verbreitung des Coronavirus werden Veranstaltungen verschoben oder fallen kurzfristig aus. Bitte prüfen Sie vorher, ob ein Kongress stattfindet.

\author{
12. Nationale Konferenz zur Versorgung \\ der Psoriasis \\ 26. Februar 2021 \\ Hamburg, Deutschland
}

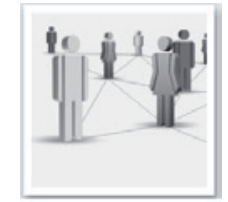

$$
\begin{array}{|l}
\text { 51. DDG-Tagung } \\
\text { 14.-17. April } 2021 \\
\text { Virtuell }
\end{array}
$$

Deutsche

Dermatologische Gesellschaft

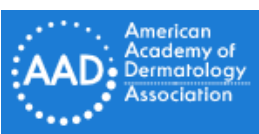

FEBRUAR 2021

4. Lübecker Update: Entzündliche Hauterkrankungen

27. Februar 2021

Lübeck, Deutschland

MÄRZ 2021

29. Jahrestagung der

Arbeitsgemeinschaft Pädiatrische

Dermatologie e. V. in der DDG

05.-06. März 2021

München, Deutschland

5th Global Summit on Dermatology

and Cosmetology

08.-09. März 2021

London, UK

APRIL 2021

10th World Congress of Melanoma in conjunction with the 17th EADO Congress

15.-17. April 2021

Virtuell

XXXVI. Jenaer Colloquium allergologicum

17. April 2021

Jena, Deutschland
MAI 2021

DEWU Deutscher Wundkongress

\& Bremer Pflegekongress

05.-07. Mai 2021

Bremen, Deutschland

7th Nordic Course on Skin Surgery 06.-07. Mai 2021

Kopenhagen, Dänemark

Allergologie im Kloster 2021

07.-08. Mai 2021

Eltville, Deutschland

JUNI 2021

6th World Congress of

Dermoscopy 2021

10.-12. Juni 2021

Buenos Aires, Argentinien

IX. Marburger Allergie-Symposion 11.-12. Juni 2021

Marburg, Deutschland

59. Dermatologisches Kolloquium der Würzburger Dermatologischen Gesellschaft (WDG)

11. Juni 2021

Würzburg, Deutschland
2. Jahresauftakt-Symposium der ADO 2021

25.-26. Juni 2021

Mainz, Deutschland

AUGUST 2021

Hautkrebs- und Systemtherapiesymposium Rhein-Ruhr 20.-21. August 2021

Köln, Deutschland

\section{SEPTEMBER 2021}

35. Jahrestagung Deutsche Gesellschaft für Dermatochirurgie

03.-05. September 2021

Hamburg, Deutschland

63. Jahrestagung der Deutschen Gesellschaft für Phlebologie 08.-11. September 2021 Aachen, Deutschland

31. Deutscher Hautkrebskongress / ADO-Jahrestagung

08.-11. September 2021

Hamburg, Deutschland 